\title{
Power Minimizer Symbol-Level Precoding: A Closed-Form Sub-Optimal Solution
}

\author{
Alireza Haqiqatnejad, Student Member, IEEE, Farbod Kayhan, and Björn Ottersten, Fellow, IEEE
}

\begin{abstract}
In this letter, we study the optimal solution of multiuser symbol-level precoding (SLP) for minimization of the total transmit power under given signal-to-interference-plusnoise ratio (SINR) constraints. Adopting the distance preserving constructive interference regions (DPCIR), we first derive a simplified reformulation of the problem. Then, we analyze the structure of the optimal solution using the Karush-Kuhn-Tucker (KKT) optimality conditions. This leads us to obtain a closed-form sub-optimal SLP solution (CF-SLP) for the original problem. Meanwhile, we obtain the necessary and sufficient condition under which the power minimizer SLP is equivalent to the conventional zero-forcing beamforming (ZFBF). Simulation results show that CF-SLP provides significant gains over ZFBF, while performing quite close to the optimal SLP in scenarios with rather small number of users. The results further indicate that the CF-SLP method has a reduction of order $10^{3}$ in computational time compared to the optimal solution.
\end{abstract}

Index Terms-Constructive interference, downlink multiuser MISO, power minimization, symbol-level precoding.

\section{INTRODUCTION}

Handling multiuser interference (MUI) is the key to increase individual reliable transmission rates of the users in a downlink multiuser channel. Extensive research focusing on multiuser transmit beamforming (precoding) with the aim of suppressing the MUI has been reported in the literature (see, e.g., [1] and the references therein). Recently, it has been shown that the MUI may not always be destructive. For example, one may try to exploit the constructive part of the interference, or even converting all the interfering components into constructive interference (CI) [2]. Subsequently, the conventional viewpoint on multiuser precoding evolved from block-level approaches (based on the channel coherence time) to more meticulous design techniques, such as symbol-level precoding (SLP) [3], [4], which results in significant performance gains. This, however, requires the use of data information (DI) in addition to channel state information (CSI) in order to design the precoder. Such a design concept enables the received components associated with the users intended signal to constructively interfere with those of the other users.

Despite the performance advantages of SLP schemes, one of the main factors that may limit their practicability is the increased complexity at the transmitter side (see [2] for an analytical discussion on the computational complexity of SLP and [5] for a possible implementation of SLP and the resulting complexity). Broadly speaking, the SLP module needs to compute the output once per symbol slot, or alternatively, one may design the precoder's output beforehand for every possible combination of users' symbols [4]. In either case, a relatively large number of optimization problems has to be solved for every realization of the time-varying

The authors are with the Interdisciplinary Centre for Security, Reliability and Trust (SnT), University of Luxembourg, L-1855 Luxembourg, Luxembourg. Email: $\{$ alireza.haqiqatnejad,farbod.kayhan,bjorn.ottersten\}@uni.lu.

The authors are supported by the Luxembourg National Research Fund (FNR) under CORE Junior project: C16/IS/11332341 Enhanced Signal Space opTImization for satellite comMunication Systems (ESSTIMS). channel. As opposed to conventional block-level approaches, e.g., optimal (objective-oriented) linear precoding [6], [7], zero-forcing beamforming (ZFBF) [8], or minimum mean-square error (MMSE) precoding [9], SLP approaches are in general highly computationally demanding. Therefore, reducing the complexity of symbol-level precoders is of great practical importance. This has been addressed in [10] for the SLP design problem with max-min fairness criterion, where an iterative closed-form method is proposed. In [11], the SLP optimization problem minimizing the total transmit power is formulated as a non-negative least squares (NNLS), which can be solved via the existing fast NNLS algorithms. For quadrature amplitude modulation (QAM) schemes, the authors in [12] analyze the structure of the optimal SLP with symbol error probability constraints and propose a heuristic low-complexity solution.

Focusing on the distance preserving CI regions (DPCIR), which are introduced in [13] and fully characterized in [14], in this letter we address the SLP power minimization problem with SINR constraints. By rearranging the original formulation and deriving a simplified version of this problem, we discuss the structure of the optimal solution via the Karush-Kuhn-Tucker (KKT) conditions. This enables us to obtain an explicit closed-form expression as a sub-optimal solution. We will show that the performance of the closed-form solution is comparable to the optimal SLP for small number of users, but with an extremely reduced complexity. Although the proposed closed-form method is more computationally complex than blocklevel precoding schemes, our results show that in return, it provides substantial gains over ZFBF and outperforms the optimal block-level precoding in [6] at high SINR thresholds. This may indeed stimulate the applicability of the proposed method in realistic scenarios.

The remainder of this letter is organized as follows. In Section II, we describe the downlink multiuser MISO model. Then, we express the distance preserving CI constraints in Section III. In Section IV, we formulate the SLP optimization problem. This is followed by providing the optimality analysis and proposing a closed-form solution. The simulation results are presented in Section V. Finally, we conclude the paper in Section VI.

Notation: To denote matrices and vectors, we use uppercase and lowercase bold-faced letters respectively, and $[\cdot]^{\mathrm{T}}$ denotes the transpose operator. For vectors, $\succeq$ denotes the componentwise inequality. blkdiag $(\cdot)$ represents a square block matrix having maindiagonal block matrices and zero off-diagonal blocks. The set of non-negative real numbers is represented by $\mathbb{R}_{+}$. And $\mathrm{E}\{\cdot\}$ denotes the expectation operator.

\section{SYSTEM MODEL}

We consider the downlink of a multiuser unicast channel where a common transmitter, equipped with $N$ antennas, sends independent data streams to $K$ single-antenna users. We confine ourselves to a scenario in which the number of users is limited by $N$, i.e., $K \leq N$ (see [15] for a complete discussion on the feasibility of power minimization subject to given SINR requirements in a multiuser channel). 
The transmitter employs a symbol-level precoder for transmission. Accordingly, at a symbol instant, the precoder maps independent data symbols $s_{1}, \ldots, s_{K}$ onto $N$ transmit antennas, with $s_{k}$ denoting the intended symbol for the $k$-th user drawn from a finite equiprobable constellation set. The signal vector to be transmitted is denoted by $\mathbf{u}=\left[u_{1}, \ldots, u_{N}\right]^{\mathrm{T}} \in \mathbb{C}^{N \times 1}$, and is a function of all the users' symbols $s_{1}, \ldots, s_{K}$. In the presence of frequency-flat fading and additive white Gaussian noise, the received signal at the receiver of the $k$-th user is

$$
r_{k}=\mathbf{h}_{k} \mathbf{u}+z_{k}, k=1, \ldots, K,
$$

where $\mathbf{h}_{k} \in \mathbb{C}^{1 \times N}$ denotes the vector of independent and identically distributed (i.i.d.) channel coefficients between $N$ transmit antennas and the single receive antenna of user $k$, and $z_{k} \sim \mathcal{C N}\left(0, \sigma_{k}^{2}\right)$ represents the complex Gaussian noise at the $k$-th receiver. It is further assumed that $\mathrm{E}\left\{\mathbf{h}_{k}^{\mathrm{H}} \mathbf{h}_{j}\right\}=\mathbf{0}, \forall k, j=1, \ldots, K, k \neq j$. The $k$-th user may optimally detect $s_{k}$ from $r_{k}$ based on the single-user maximum-likelihood (ML) decision rule. In the following, we adopt the equivalent real-valued notations

$$
\tilde{\mathbf{u}}=\left[\Re\left\{\mathbf{u}^{\mathrm{T}}\right\}, \Im\left\{\mathbf{u}^{\mathrm{T}}\right\}\right]^{\mathrm{T}}, \mathbf{H}_{k}=\left[\begin{array}{lr}
\Re\left\{\mathbf{h}_{k}\right\} & -\Im\left\{\mathbf{h}_{k}\right\} \\
\Im\left\{\mathbf{h}_{k}\right\} & \Re\left\{\mathbf{h}_{k}\right\}
\end{array}\right],
$$

where $\tilde{\mathbf{u}} \in \mathbb{R}^{2 N \times 1}$ and $\mathbf{H}_{k} \in \mathbb{R}^{2 \times 2 N}, k=1, \ldots, K$. Henceforth, the user's noise-free received signal is represented by $\mathbf{H}_{k} \tilde{\mathbf{u}}=\left[\Re\left\{\mathbf{h}_{k} \mathbf{u}\right\}, \Im\left\{\mathbf{h}_{k} \mathbf{u}\right\}\right]^{\mathrm{T}}$ for all $k=1, \ldots, K$.

\section{CONSTRUCtive InTERFEREnCE CONSTRAints}

The DI exploitation in symbol-level design can be realized by processing the transmit signal in order to have the (noise-free) received signal of each user in a specific CIR associated with the desired symbol. CIRs are generally defined such that they preserve or enhance the symbol detection accuracy with respect to (w.r.t.) the original constellation set [13], [16]. In [13], DPCIRs have been introduced as a general family of CIRs that preserve the Euclidean distances between the constellation points, i.e., they do not increase the symbol error probabilities of the users. The halfspace representation of DPCIRs is provided for generic modulation schemes based on the ML decision regions of the constellation set.

For a constellation point $\mathbf{x}_{i}$, the corresponding DPCIR is the intersection of a finite number of halfspaces, each associated with one of $M_{i}$ neighboring points of $\mathbf{x}_{i}$ (the neighboring points are referred to those points sharing a decision boundary with $\mathbf{x}_{i}$ ). More specifically, using the representation provided in [13], it is straightforward to show that any point $\mathbf{x}$ in the DPCIR of $\mathbf{x}_{i}$ satisfies a linear matrix inequality (LMI) as

$$
\mathbf{A}_{i}\left(\mathbf{x}-\mathbf{x}_{i}\right) \succeq \mathbf{0}
$$

where $\mathbf{A}_{i} \in \mathbb{R}^{M_{i} \times 2}$ is a matrix that contains the normal vectors of DPCIR boundaries (hyperplanes), given by

$$
\mathbf{A}_{i}=\left[\begin{array}{c}
\mathbf{a}_{i, 1}^{\mathrm{T}} \\
\vdots \\
\mathbf{a}_{i, M_{i}}^{\mathrm{T}}
\end{array}\right]=\left[\begin{array}{c}
\left(\mathbf{x}_{i}-\mathbf{x}_{i, 1}\right)^{\mathrm{T}} \\
\vdots \\
\left(\mathbf{x}_{i}-\mathbf{x}_{i, M_{i}}\right)^{\mathrm{T}}
\end{array}\right],
$$

with $\mathbf{x}_{i, 1}, \ldots, \mathbf{x}_{i, M_{i}}$ denoting the neighboring constellation points of $\mathbf{x}_{i}$. Introducing a non-negative vector $\boldsymbol{\delta}_{i} \in \mathbb{R}_{+}^{M_{i} \times 1}$, the LMI in (2) is equivalent to

$$
\mathbf{A}_{i}\left(\mathbf{x}-\mathbf{x}_{i}\right)=\boldsymbol{\delta}_{i}, \boldsymbol{\delta}_{i} \succeq \mathbf{0},
$$

which will be used as the CI constraint in our formulation of the SLP optimization problem. It is worth noting that the entries of $\boldsymbol{\delta}_{i}$ are proportional to the orthogonal distances between $\mathbf{x}$ and the DPCIR boundaries of $\mathbf{x}_{i}$. A complete characterization of DPCIRs along with the geometric interpretation of $\boldsymbol{\delta}_{i}$ is provided in [14] and [17] It is shown that if $\mathbf{x}_{i}$ is a constellation point with bounded decision region, we always have $\boldsymbol{\delta}_{i}=\mathbf{0}$. On the other hand, if $\mathbf{x}_{i}$ refers to a constellation point with unbounded decision region, the associated DPCIR is a polyhedral angle with two infinite edges starting from $\mathbf{x}_{i}$. Hence, there always exist exactly two halfspaces that are sufficient to characterize the DPCIR, and to construct $\mathbf{A}_{i} \in \mathbb{R}^{2 \times 2}$ as a nonsingular matrix. Furthermore, any point $\mathbf{x}$ that satisfies (4) can be uniquely represented by $\boldsymbol{\delta}_{i} \in \mathbb{R}_{+}^{2 \times 1}$. For more details on characterizing the DPCIRs, the readers are kindly referred to [14] and [17].

\section{SINR-CONSTRAINED POWER MINIMIZER SLP}

In this section, we are interested in the symbol-level power minimization problem being constrained by CIRs as well as individual SINR requirements, i.e.,

$$
\begin{aligned}
\underset{\mathbf{u}}{\operatorname{minimize}} & \mathbf{u}^{\mathrm{T}} \mathbf{u} \\
\text { s.t. } & \mathbf{h}_{k} \mathbf{u} \in \sigma_{k} \sqrt{\gamma_{k}} \mathcal{D}_{k}, k=1, \ldots, K,
\end{aligned}
$$

where $\gamma_{k}$ and $\mathcal{D}_{k}$ respectively denote the required SINR and the CIR related to the $k$-th user. By assuming DPCIRs, the CI expressions in the form of (4) can be used to explicitly define the CI constraints of (5).

In order to simplify the analysis and notation, and without loss of generality, we assume an identical $M$-ary constellation set $\mathcal{X}=\left\{\mathbf{x}_{i} \mid \mathbf{x}_{i} \in \mathbb{R}^{2 \times 1}\right\}_{i=1}^{M}$ with unit average power for all $K$ users. A symbol $s_{k}$ then corresponds to one of the points $\left\{\mathbf{x}_{i}\right\}_{i=1}^{M}$ in $\mathcal{X}$. We denote by $i_{k}$ the index of the constellation point corresponding to $s_{k}$, i.e.,

$$
\mathbf{x}_{i_{k}}=\left[\Re\left\{s_{k}\right\}, \Im\left\{s_{k}\right\}\right]^{\mathrm{T}}, i_{k} \in\{1, \ldots, M\}, k=1, \ldots, K .
$$

In the rest, we consider the case in which all $\mathbf{x}_{i_{k}}$ have unbounded decision regions, i.e., $\boldsymbol{\delta}_{i_{k}} \in \mathbb{R}_{+}^{2 \times 1}, k=1, \ldots, K$. It is straightforward to generalize the results to the case with the users' symbols comprising a mix of points with both bounded and unbounded decision regions.

For each user $k, \mathbf{H}_{k} \tilde{\mathbf{u}}$ is pushed by the precoder to be inside the corresponding DPCIR up to a scale factor that depends on the given SINR requirement. From (4), by substituting $\mathbf{H}_{k} \tilde{\mathbf{u}}$ for $\mathbf{x}$ and replacing the scaled constellation point $\sigma_{k} \sqrt{\gamma_{k}} \mathbf{x}_{i_{k}}$, we obtain the CI constraint for the $k$-th user as

$$
\mathbf{A}_{i_{k}}\left(\mathbf{H}_{k} \tilde{\mathbf{u}}-\sigma_{k} \sqrt{\gamma_{k}} \mathbf{x}_{i_{k}}\right)=\boldsymbol{\delta}_{i_{k}}, \boldsymbol{\delta}_{i_{k}} \succeq \mathbf{0} .
$$

Taking all the users into account, by stacking the CI constraint (6) for all $k \in\{1, \ldots, K\}$ into the matrix form, we have

$$
\mathbf{A}\left(\tilde{\mathbf{H}} \tilde{\mathbf{u}}-\boldsymbol{\Sigma} \boldsymbol{\Gamma}^{1 / 2} \tilde{\mathbf{x}}\right)=\boldsymbol{\delta}, \boldsymbol{\delta} \succeq \mathbf{0},
$$

where $\tilde{\mathbf{H}} \triangleq\left[\mathbf{H}_{1}^{\mathrm{T}}, \ldots, \mathbf{H}_{K}^{\mathrm{T}}\right]^{\mathrm{T}}, \mathbf{A} \triangleq \operatorname{blkdiag}\left(\mathbf{A}_{i_{1}}, \ldots, \mathbf{A}_{i_{K}}\right)$, $\boldsymbol{\Sigma} \triangleq \operatorname{blkdiag}\left(\sigma_{1}, \ldots, \sigma_{K}\right), \boldsymbol{\Gamma} \triangleq \operatorname{blkdiag}\left(\gamma_{1}, \ldots, \gamma_{K}\right)$, $\tilde{\mathbf{x}} \triangleq\left[\mathbf{x}_{i_{1}}, \ldots, \mathbf{x}_{i_{K}}\right]^{\mathrm{T}}$, and $\boldsymbol{\delta} \triangleq\left[\boldsymbol{\delta}_{i_{1}}, \ldots, \boldsymbol{\delta}_{i_{K}}\right]^{\mathrm{T}}$. Recalling that all $\mathbf{A}_{i_{k}}, k=1, \ldots, K$ are non-singular, it can be verified that $\mathbf{A}$ is invertible. Therefore, (7) can be written as

$$
\tilde{\mathbf{H}} \tilde{\mathbf{u}}=\boldsymbol{\Sigma} \boldsymbol{\Gamma}^{1 / 2} \tilde{\mathbf{x}}+\mathbf{A}^{-1} \boldsymbol{\delta}, \boldsymbol{\delta} \succeq \mathbf{0} .
$$

Using (8), a (convex) compact formulation for the optimization problem of interest can be expressed as

$$
\begin{aligned}
\underset{\tilde{\mathbf{u}}, \boldsymbol{\delta} \succeq \mathbf{0}}{\operatorname{minimize}} & \tilde{\mathbf{u}}^{\mathrm{T}} \tilde{\mathbf{u}} \\
\text { s.t. } & \tilde{\mathbf{H}} \tilde{\mathbf{u}}=\boldsymbol{\Sigma} \boldsymbol{\Gamma}^{1 / 2} \tilde{\mathbf{x}}+\mathbf{A}^{-1} \boldsymbol{\delta} .
\end{aligned}
$$

We further notice that $\tilde{\mathbf{H}}$ is (almost surely) a full row rank matrix due to its stochastic nature. This results in a bijection between $\tilde{\mathbf{u}}$ and $\boldsymbol{\delta}$ in (8), i.e., for any given $\boldsymbol{\delta}$, the (least-norm) $\tilde{\mathbf{u}}$ is obtained by 


$$
\underset{\tilde{\mathbf{u}}}{\operatorname{argmin}} \tilde{\mathbf{u}}^{\mathrm{T}} \tilde{\mathbf{u}} \triangleq \tilde{\mathbf{u}}_{\mathrm{LN}}(\boldsymbol{\delta})=\underbrace{\tilde{\mathbf{H}}^{\dagger} \boldsymbol{\Sigma} \boldsymbol{\Gamma}^{1 / 2} \tilde{\mathbf{x}}}_{\tilde{\mathbf{u}}_{\mathrm{ZF}}}+\underbrace{\tilde{\mathbf{H}}^{\dagger} \mathbf{A}^{-1} \boldsymbol{\delta}}_{\tilde{\mathbf{u}}_{\mathrm{SL}}},
$$

where $\tilde{\mathbf{H}}^{\dagger}=\tilde{\mathbf{H}}^{\mathrm{T}}\left(\tilde{\mathbf{H}} \tilde{\mathbf{H}}^{\mathrm{T}}\right)^{-1}$ is the Moore-Penrose inverse of $\tilde{\mathbf{H}}$. Equation (10) gives the structure of the power minimizer transmit vector, i.e., the optimal solution of (9). Intuitively, it consists of two parts: $\tilde{\mathbf{u}}_{\mathrm{ZF}}$, which is the solution of ZFBF, and $\tilde{\mathbf{u}}_{\mathrm{SL}}$, the DPCIRdependent part which brings the (potential) gain of SLP with respect to ZFBF. Accordingly, the optimization problem (9) reduces to

$$
\boldsymbol{\delta}^{*}=\underset{\boldsymbol{\delta} \succeq \mathbf{0}}{\operatorname{argmin}}\left\|\tilde{\mathbf{H}}^{\dagger} \boldsymbol{\Sigma} \boldsymbol{\Gamma}^{1 / 2} \tilde{\mathbf{x}}+\tilde{\mathbf{H}}^{\dagger} \mathbf{A}^{-1} \boldsymbol{\delta}\right\|^{2} .
$$

The optimal transmit vector is then obtained by

$$
\tilde{\mathbf{u}}^{*}=\tilde{\mathbf{u}}_{\mathrm{LN}}\left(\boldsymbol{\delta}^{*}\right) .
$$

Problem (11) is classified as a quadratic programming (QP) [18]. There are various algorithms to solve a QP optimization, e.g., interior-point or active set methods and gradient algorithms [19]; however, they typically require a rather large number of iterations to converge. This motivates us to find a more tractable, possibly sub-optimal, solution for (11).

1) Optimality Analysis: The Lagrangian of (11) is given by

$$
\begin{aligned}
\mathcal{L}(\boldsymbol{\delta}, \boldsymbol{\lambda}) & =\tilde{\mathbf{x}}^{\mathrm{T}} \boldsymbol{\Sigma} \boldsymbol{\Gamma}^{1 / 2} \tilde{\mathbf{H}}^{\dagger \mathrm{T}} \tilde{\mathbf{H}} \boldsymbol{\Sigma} \boldsymbol{\Gamma}^{1 / 2} \tilde{\mathbf{x}} \\
& +\boldsymbol{\delta}^{\mathrm{T}} \mathbf{A}^{-\mathrm{T}} \tilde{\mathbf{H}}^{\dagger \mathrm{T}} \tilde{\mathbf{H}}^{\dagger} \boldsymbol{\Sigma} \boldsymbol{\Gamma}^{1 / 2} \tilde{\mathbf{x}}+\tilde{\mathbf{x}}^{\mathrm{T}} \boldsymbol{\Sigma} \boldsymbol{\Gamma}^{1 / 2} \tilde{\mathbf{H}}^{\dagger \mathrm{T}} \tilde{\mathbf{H}}^{\dagger} \mathbf{A}^{-1} \boldsymbol{\delta} \\
& +\boldsymbol{\delta}^{\mathrm{T}} \mathbf{A}^{-\mathrm{T}} \tilde{\mathbf{H}}^{\dagger \mathrm{T}} \tilde{\mathbf{H}}^{\dagger} \mathbf{A}^{-1} \boldsymbol{\delta}+\boldsymbol{\lambda}^{\mathrm{T}} \boldsymbol{\delta},
\end{aligned}
$$

from which the Lagrange dual problem can be written as

$$
\max _{\boldsymbol{\lambda} \preceq \mathbf{0}} \inf _{\boldsymbol{\delta} \succeq \mathbf{0}} \mathcal{L}(\boldsymbol{\delta}, \boldsymbol{\lambda}),
$$

where $\lambda$ is the vector of the Lagrange multipliers. Denoting the primal and dual optimals by $\delta^{*}$ and $\lambda^{*}$, respectively, the Karush-Kuhn-Tucker (KKT) optimality conditions are

$$
\begin{array}{rlrl}
\nabla_{\boldsymbol{\delta}} \mathcal{L}\left(\boldsymbol{\delta}^{*}, \boldsymbol{\lambda}^{*}\right) & =\mathbf{0}, & & \text { (stationarity) } \\
\boldsymbol{\delta}^{*} \succeq \mathbf{0}, & & \text { (primal feasibility) } \\
\boldsymbol{\lambda}^{*} \preceq \mathbf{0}, & & \text { (dual feasibility) } \\
\boldsymbol{\lambda}^{* \mathrm{~T}} \boldsymbol{\delta}^{*}=0, & & \text { (complementary slackness) }
\end{array}
$$

Notice that since the primal problem (11) is convex, strong duality holds and the KKT conditions (14a)-(14d) are necessary and sufficient [18]. Consequently, a candidate solution satisfying all the KKT conditions is globally optimal.

Let $\mathbf{Q}=\mathbf{Q}^{\mathrm{T}} \triangleq \mathbf{A}^{-\mathrm{T}} \tilde{\mathbf{H}}^{\dagger \mathrm{T}} \tilde{\mathbf{H}}^{\dagger} \mathbf{A}^{-1}=\left[\mathbf{q}_{1}, \ldots, \mathbf{q}_{2 K}\right]^{\mathrm{T}}$ and $\mathbf{v} \triangleq \mathbf{A}^{-\mathrm{T}} \tilde{\mathbf{H}}^{\dagger \mathrm{T}} \tilde{\mathbf{H}}^{\dagger} \boldsymbol{\Sigma} \boldsymbol{\Gamma}^{1 / 2} \tilde{\mathbf{x}}=\left[v_{1}, \ldots, v_{2 K}\right]^{\mathrm{T}}$. The stationarity condition (14a) is derived as $2 \mathbf{Q} \boldsymbol{\delta}^{*}+2 \mathbf{v}+\boldsymbol{\lambda}^{*}=\mathbf{0}$ and thus,

$$
\lambda^{*}=-2\left(\mathbf{Q} \boldsymbol{\delta}^{*}+\mathbf{v}\right) \text {. }
$$

It then follows from (14c) and (15) that

$$
\mathbf{Q} \delta^{*}+\mathbf{v} \succeq \mathbf{0} .
$$

Furthermore, substituting $\boldsymbol{\lambda}^{*}$ from (15) in (14d) yields

$$
\left(\mathbf{Q} \boldsymbol{\delta}^{*}+\mathbf{v}\right)^{\mathrm{T}} \boldsymbol{\delta}^{*}=0,
$$

from which by denoting $\boldsymbol{\psi} \triangleq \mathbf{Q} \boldsymbol{\delta}^{*}+\mathbf{v}=\left[\psi_{1}, \ldots, \psi_{2 K}\right]^{\mathrm{T}}$ and $\delta^{*}=\left[\delta_{1}^{*}, \ldots, \delta_{2 K}^{*}\right]^{\mathrm{T}}$, it follows that

$$
\sum_{l=1}^{2 K} \psi_{l} \delta_{l}^{*}=0 .
$$

Considering (14b) and (16), we have $\psi_{l} \geq 0, l=1, \ldots, 2 K$. As a consequence, the optimality condition (18) is met iff

$$
\psi_{l} \delta_{l}^{*}=0, \forall l \in\{1, \ldots, 2 K\} .
$$

In other words, $\psi_{l}$ and $\delta_{l}^{*}$ cannot be both non-zero for any specific $l \in\{1, \ldots, 2 K\}$. Based on this observation, the following lemma relates the SLP solution to that of ZFBF.

Lemma 1. The optimal solution of the SLP power minimization (9) is identical to the solution of ZFBF iff $\mathbf{v} \succeq \mathbf{0}$.

Proof. Sufficiency: It is clear from (10) and (12) that $\tilde{\mathbf{u}}^{*}$ equals the solution of ZFBF iff $\boldsymbol{\delta}^{*}=\mathbf{0}$. Given $\mathbf{v} \succeq \mathbf{0}$, let assume by contradiction that $\boldsymbol{\delta}^{*} \neq \mathbf{0}$, i.e., $\exists l$ such that $\delta_{l}^{*}>0$, which gives $\mathbf{v}^{\mathrm{T}} \boldsymbol{\delta}^{*} \geq 0$. Let us rewrite the optimality condition (17) as $\boldsymbol{\delta}^{* \mathrm{~T}} \mathbf{Q} \boldsymbol{\delta}^{*}+\mathbf{v}^{\mathrm{T}} \boldsymbol{\delta}^{*}=0$. By definition, $\mathbf{Q}$ is symmetric and $\mathbf{Q}=\left(\tilde{\mathbf{H}}^{\dagger} \mathbf{A}^{-1}\right)^{\mathrm{T}} \tilde{\mathbf{H}}^{\dagger} \mathbf{A}^{-1}$, where $\tilde{\mathbf{H}}^{\dagger} \mathbf{A}^{-1}$ has full column rank due to the random concatenated channel $\tilde{\mathbf{H}}$. Hence, $\mathbf{Q}$ is a positive definite matrix [20, Theorem 7.2.7], i.e., $\boldsymbol{\delta}^{* \mathrm{~T}} \mathbf{Q} \boldsymbol{\delta}^{*}>0$ for any $\boldsymbol{\delta}^{*} \neq \mathbf{0}$. This, however, yields $\boldsymbol{\delta}^{* \mathrm{~T}} \mathbf{Q} \boldsymbol{\delta}^{*}+\mathbf{v}^{\mathrm{T}} \boldsymbol{\delta}^{*}>0$ which contradicts the KKT condition (17). Therefore, having $\mathbf{v} \succeq \mathbf{0}$, it necessarily holds that $\delta^{*}=\mathbf{0}$, as required.

Necessity: Assuming $\boldsymbol{\delta}^{*}=\mathbf{0}$, it immediately follows from (16) that $\mathbf{v} \succeq \mathbf{0}$. This completes the proof.

Lemma 1 provides the necessary and sufficient condition under which the DPCIR-based SLP has the same solution as ZFBF. This occurs depending on the instantaneous realization of the users' symbols as $\mathbf{v} \succeq \mathbf{0}$ is equivalently met by $\mathbf{A}^{-\mathrm{T}} \tilde{\mathbf{H}}^{\dagger \mathrm{T}} \tilde{\mathbf{H}}^{\dagger} \boldsymbol{\Sigma} \boldsymbol{\Gamma}^{1 / 2} \tilde{\mathbf{x}} \succeq \mathbf{0}$. It can be further inferred from (19) and Lemma 1 that as the number of non-zero (i.e. positive) elements of $\psi$ decreases, the SLP solution may diverge from that of the ZFBF. In the extreme case with $\psi_{l}=0, l=1, \ldots, 2 K$, there exists at least one $\delta_{l}^{*} \neq 0$. It can be verified as $\psi=\mathbf{Q} \delta^{*}+\mathbf{v}=\mathbf{0}$ has a unique solution equal to $\delta^{*}=-\mathbf{Q}^{-1} \mathbf{v}=$ $\mathbf{A} \boldsymbol{\Sigma} \boldsymbol{\Gamma}^{1 / 2} \tilde{\mathbf{x}}$. Since $\mathbf{A}$ is full rank, it has an empty null space, thus $\mathbf{A} \boldsymbol{\Sigma} \boldsymbol{\Gamma}^{1 / 2} \tilde{\mathbf{x}} \neq \mathbf{0}$. This means that $\boldsymbol{\delta}^{*} \neq \mathbf{0}$ and it has at least one nonzero entry. In such case, the SLP design results in higher precoding gains compared to the ZFBF. This case, however, is feasible only if the unique solution to the system of linear equations $\mathbf{Q} \delta^{*}+\mathbf{v}=\mathbf{0}$ is non-negative, i.e., $-\mathbf{Q}^{-1} \mathbf{v} \succeq \mathbf{0}$, or equivalently $\mathbf{A} \boldsymbol{\Sigma} \boldsymbol{\Gamma}^{1 / 2} \tilde{\mathbf{x}} \preceq \mathbf{0}$.

2) Closed-Form Sub-Optimal Solution: By utilizing the KKT optimality analysis, a low-complexity solution can be derived with a simple idea behind. Let $\mathcal{Z} \triangleq\left\{l \mid \delta_{l}^{*} \neq 0\right\}$ be the set of inactive constraints of (11) at the optimum, then according to (19), we have

$$
\psi_{l}=\mathbf{q}_{l}^{\mathrm{T}} \boldsymbol{\delta}^{*}+v_{l}=0, \forall l \in \mathcal{Z} .
$$

which gives a reduced system of linear equations to obtain $\delta^{*}$. However, in theory, we do not have such prior information, i.e., the inactive set $\mathcal{Z}$ is not explicitly known. Instead, we can approximate $\mathcal{Z}$ as follows. From (17), the positive definiteness of $\mathbf{Q}$ verifies that

$$
\mathbf{v}^{\mathrm{T}} \boldsymbol{\delta}^{*}=\sum_{l=1}^{2 K} v_{l} \delta_{l}^{*} \leq 0
$$

where equality holds only for $\boldsymbol{\delta}^{*}=\mathbf{0}$. An approximation of $\mathcal{Z}$ can be derived based on the sign of the elements in $\mathbf{v}$, i.e., $\hat{\mathcal{Z}}=\left\{l \mid v_{l}<0\right\}$ with $|\hat{\mathcal{Z}}|=L$. Here, it is assumed that $\delta_{l}^{*}=0$ (i.e., the $l$-th constraint is active at the optimum) for those $l$ with $v_{l} \geq 0$. This results in a reduced system of linear equations given by

$$
\mathbf{Q}^{\prime} \boldsymbol{\delta}^{* \prime}+\mathbf{v}^{\prime}=\mathbf{0},
$$

where $\mathbf{Q}^{\prime} \in \mathbb{R}^{L \times L}, \boldsymbol{\delta}^{* \prime} \in \mathbb{R}^{L \times 1}$ and $\mathbf{v}^{\prime} \in \mathbb{R}^{L \times 1}$ are punctured versions of $\mathbf{Q}, \boldsymbol{\delta}^{*}$ and $\mathbf{v}$ obtained by excluding the equations, variables and coefficients corresponding to $l \notin \hat{\mathcal{Z}}$. This new system has $L$ linear equations and $L$ variables, where $L \leq 2 K$, hence possibly less 

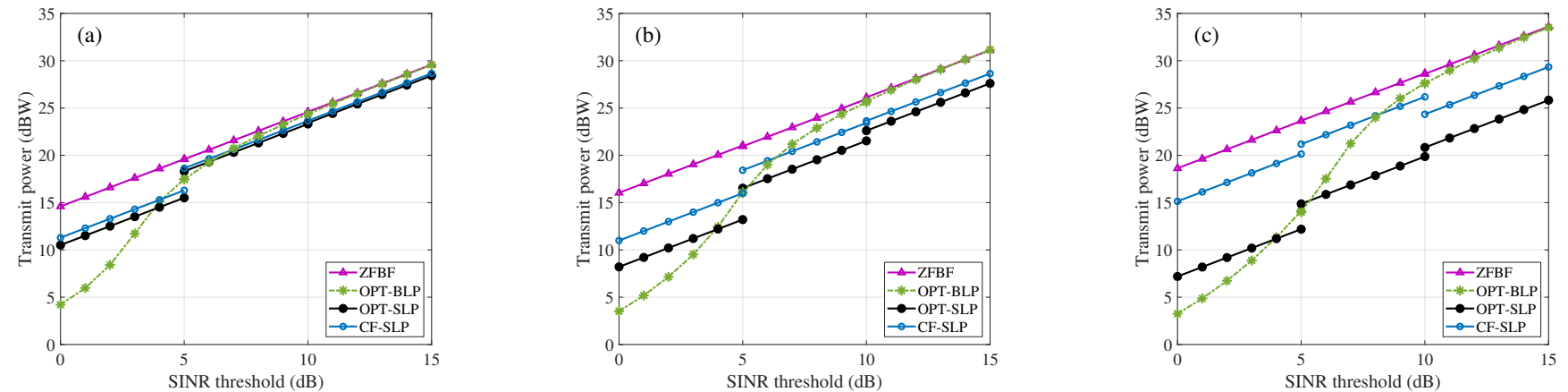

Fig. 1. Total transmit power versus SINR threshold for different system dimensions (a) $4 \times 4$ (b) $8 \times 8$ (c) $16 \times 16$.

dimensionality than the original problem. By noticing that the nonsingularity of $\mathbf{Q}$ is preserved under puncturing, the (unique) solution of (22) is readily given by the following closed-form expression:

$$
\boldsymbol{\delta}^{* \prime}=\max \left\{-\mathbf{Q}^{\prime-1} \mathbf{v}^{\prime}, \mathbf{0}\right\},
$$

where $\max \{\cdot\}$ denotes the elementwise maximum, and is applied in order to guarantee the primal feasibility condition (14b). The entire $\delta^{*}$ can be obtained by inserting the zero entries $\delta_{l}^{*}, l \notin \hat{\mathcal{Z}}$ into $\delta^{* \prime}$. The resulting $\delta^{*}$ is then a sub-optimal solution for (11).

The proposed method can be easily generalized to the case where the users' symbols can also be taken from interior constellation points. In such case, similar analysis holds through multiplying $\delta$ by a diagonal binary weighting matrix $\mathbf{W}$ in (9). Details on how to construct $\mathbf{W}$ can be found in [17].

\section{Simulation Results}

In this section, we evaluate the performance of the proposed closed-form solution (CF-SLP). The results are compared with those of the ZFBF, the optimal power minimizer block-level (OPT-BLP) [6] and the optimal power minimizer symbol-level (OPT-SLP) precoding schemes. We assume equal SINR thresholds, i.e., $\gamma_{k}=\gamma, k=$ $1, \ldots, K$, and only focus on scenarios with $N=K$. The complex channel vectors are randomly generated as $\mathbf{h}_{k} \sim \mathcal{C N}(\mathbf{0}, \mathbf{I}), k=1, \ldots, K$. All the simulations are done using MATLAB software and CVX convex optimization package [21] (SDPT3 solver).

In Fig. 1, we plot the total transmit power as a function of SINR threshold for three system dimensions with $K=4,8$ and 16. In the depicted range of SINR, three different modulation schemes, namely, QPSK, optimized 8-ary [13], [22], and 16-QAM, are respectively employed in the intervals $0-5,5-10$ and $10-15 \mathrm{~dB}$. As it can be observed, in $4 \times 4$ scenario, CF-SLP is almost equally as power-efficient as OPT-SLP. The loss due to sub-optimality of CF-SLP is not significant, with a maximum loss of $0.8 \mathrm{dBW}$ for QPSK. This loss is larger for $8 \times 8$ and $16 \times 16$ system dimensions, as shown in Fig. 1 (b) and Fig. 1 (c). This can be explained by the possibility of having more errors in $\hat{\mathcal{Z}}$ (w.r.t. $\mathcal{Z}$ ) as the problem size increases. Notice, however, that SLP shows higher performance gains for larger system dimensions, and CF-SLP offers considerable gains up to $5 \mathrm{dBW}$ compared to ZFBF for large system dimensions. At low SINR thresholds, OPT-BLP is the most power-efficient scheme, but in fact this reduction in the transmit power is obtained at the cost of a degraded symbol error rate (SER) (see [13] for related discussions). Nevertheless, the OPT-BLP scheme approaches ZFBF as the SINR threshold increases.

In Table I, we compare the complexity in terms of the average execution time per symbol slot (the time values are obtained via the relevant functions of MATLAB and CVX). As for the ZFBF and OPT-BLP schemes, the precoding matrix is multiplied by the users' symbol vector every symbol time. The precoding matrix computation, which is typically updated as frequent as the channel coherence time, is also accounted for in the per-symbol execution times (assuming 100 symbols within each coherence time). The CF-SLP method consists of computing and puncturing $\mathbf{Q}$ and $\mathbf{v}$, and then solving (23). On the other hand, solving the convex power optimization (11) accounts for the execution time of OPT-SLP. The numerical results show that CF-SLP can potentially reduce the complexity of the SLP precoding module by orders of $10^{3}$. The execution times of CF-SLP are expectedly greater (by orders of 10) in comparison with ZFBF, but are comparable to those of OPT-BLP. This indicates a performance-complexity tradeoff, particularly for large system dimensions.

TABLE I

EXECUTION TIME OF THE PRECODING SCHEMES.

\begin{tabular}{cccccc}
\hline Modulation & Dimension & \multicolumn{4}{c}{ Execution time (ms/symbol) } \\
\cline { 3 - 6 } & & ZFBF & OPT-BLP & OPT-SLP & CF-SLP \\
\hline QPSK & $4 \times 4$ & 0.0064 & 0.0249 & 573.8417 & 0.0671 \\
& $8 \times 8$ & 0.0076 & 0.0779 & 537.4583 & 0.1222 \\
& $16 \times 16$ & 0.0113 & 0.3098 & 595.9375 & 0.2388 \\
\hline Optimized 8-ary & $4 \times 4$ & 0.0060 & 0.0215 & 588.1708 & 0.0633 \\
& $8 \times 8$ & 0.0080 & 0.0771 & 532.4833 & 0.1217 \\
& $16 \times 16$ & 0.0114 & 0.3627 & 584.1917 & 0.2223 \\
\hline 16-QAM & $4 \times 4$ & 0.0058 & 0.0194 & 554.3417 & 0.0582 \\
& $8 \times 8$ & 0.0080 & 0.0641 & 533.9958 & 0.1139 \\
& $16 \times 16$ & 0.0097 & 0.2586 & 518.7500 & 0.1760 \\
\hline
\end{tabular}

\section{CONCLUSION}

In this letter, we propose a closed-form sub-optimal solution for the SINR-constrained power minimizer SLP. This is done by first simplifying the original formulation, and then discussing the optimality of the reduced problem via the KKT conditions. Through the optimality analysis, we obtain the necessary and sufficient condition under which the power minimizer SLP has the same solution as ZFBF. The analysis further helps us to derive a closed-form sub-optimal SLP (CF-SLP). In particular, for scenarios with rather small number of users, CF-SLP offers quite similar performance compared to the computationally demanding optimal SLP. In comparison with block-level precoding approaches, our results show that CF-SLP always outperforms the conventional ZFBF, whereas at low SINR thresholds, the optimal power minimizer block-level precoding has a superior performance in terms of power-efficiency. The results suggest that CF-SLP can be an appropriate alternative (with a comparable complexity) for block-level precoding, especially in high SINR regimes. 


\section{REFERENCES}

[1] A. B. Gershman, N. D. Sidiropoulos, S. Shahbazpanahi, M. Bengtsson, and B. Ottersten, "Convex optimization-based beamforming," IEEE Signal Process. Mag., vol. 27, no. 3, pp. 62-75, May 2010.

[2] C. Masouros, "Correlation rotation linear precoding for MIMO broadcast communications," IEEE Trans. Signal Process., vol. 59, no. 1, pp. 252-262, Jan. 2011.

[3] C. Masouros and G. Zheng, "Exploiting known interference as green signal power for downlink beamforming optimization," IEEE Trans. Signal Process., vol. 63, no. 14, pp. 3628-3640, Jul. 2015.

[4] M. Alodeh, S. Chatzinotas, and B. Ottersten, "Constructive multiuser interference in symbol level precoding for the MISO downlink channel," IEEE Trans. Signal Process., vol. 63, no. 9, pp. 2239-2252, May 2015.

[5] M. Alodeh, D. Spano, A. Kalantari, C. Tsinos, D. Christopoulos, S. Chatzinotas, and B. Ottersten, "Symbol-level and multicast precoding for multiuser multiantenna downlink: A state-of-the-art, classification and challenges," IEEE Commun. Surveys Tutorials, pp. 1-1, 2018.

[6] M. Bengtsson and B. Ottersten, Handbook of Antennas in Wireless Communications, 2001, ch. Optimal and suboptimal transmit beamforming.

[7] M. Schubert and H. Boche, "Solution of the multiuser downlink beamforming problem with individual SINR constraints," IEEE Trans. Veh. Technol., vol. 53 , no. 1, pp. 18-28, Jan. 2004.

[8] T. Yoo and A. Goldsmith, "On the optimality of multiantenna broadcast scheduling using zero-forcing beamforming," IEEE J. Sel. Areas in Commun., vol. 24, no. 3, pp. 528-541, Mar. 2006.

[9] S. M. Kay, Fundamentals of Statistical Signal Processing: Estimation Theory. Upper Saddle River, NJ, USA: Prentice-Hall, Inc., 1993.

[10] A. Li and C. Masouros, "Interference exploitation precoding made practical: Closed-form solutions with optimal performance," arXiv:1712.07846 [cs.IT], Dec. 2017.

[11] J. Krivochiza, A. Kalantari, S. Chatzinotas, and B. Ottersten, "Low complexity symbol-level design for linear precoding systems," in Symposium on Information Theory and Signal Processing in the Benelux, Mar. 2016.

[12] C. Masouros, M. Sellathurai, and T. Ratnarajah, "Vector perturbation based on symbol scaling for limited feedback MISO downlinks," IEEE Trans. Signal Process., vol. 62, no. 3, pp. 562-571, Feb. 2014.

[13] A. Haqiqatnejad, F. Kayhan, and B. Ottersten, "Constructive interference for generic constellations,” IEEE Signal Process. Lett., vol. 25, no. 4, pp. 586-590, Apr. 2018.

[14] — "Symbol-level precoding design for max-min SINR in multiuser MISO broadcast channels," in 2018 IEEE 19th International Workshop on Signal Processing Advances in Wireless Communications (SPAWC), Jun. 2018.

[15] R. Hunger and M. Joham, "A complete description of the QoS feasibility region in the vector broadcast channel," IEEE Trans. Signal Process., vol. 58, no. 7, pp. 3870-3878, Jul. 2010.

[16] C. Masouros, T. Ratnarajah, M. Sellathurai, C. B. Papadias, and A. K. Shukla, "Known interference in the cellular downlink: a performance limiting factor or a source of green signal power?" IEEE Commun. Mag., vol. 51, no. 10, pp. 162-171, Oct. 2013.

[17] A. Haqiqatnejad, F. Kayhan, and B. Ottersten, "Symbol-level precoding design based on distance preserving constructive interference regions," arXiv:1804.00930 [eess.SP], Mar. 2018.

[18] S. Boyd and L. Vandenberghe, Convex Optimization. Cambridge Univ. Press, 2004.

[19] R. M. Freund, "Solution methods for quadratic optimization," 2004.

[20] R. A. Horn and C. R. Johnson, Matrix analysis. Cambridge university press, 1990.

[21] CVX: MATLAB software for disciplined convex programming. [Online]. Available: http://cvxr.com/cvx

[22] F. Kayhan and G. Montorsi, "Constellation design for memoryless phase noise channels," IEEE Trans. Wirel. Commun., vol. 13, no. 5, pp. 2874-2883, May 2014. 